\title{
Chiral Power Change upon \\ Photoisomerization in Twisted Nematic Liquid Crystals
}

Sabrina Simoncelli ${ }^{a, b}$ and Pedro F. Aramendía ${ }^{a, b^{*}}$

${ }^{a}$ INQUIMAE and Departamento de Química Inorgánica, Analítica y Química Física. Facultad de Ciencias Exactas y Naturales, Universidad de Buenos Aires, Pabellón 2, Ciudad Universitaria. 1428 Ciudad de Buenos Aires, Argentina.

${ }^{\mathrm{b}}$ CIBION. Centro de Investigaciones en Bionanociencias "Elizabeth Jares-Erijman”. Godoy Cruz 2390. 1425 Ciudad de Buenos Aires. Argentina.

* Correspondence author: pedro.aramendia@cibion.conicet.gov.ar

Sabrina Simoncelli: sabrina.simoncelli@gmail.com

RECEIVED DATE (to be automatically inserted after your manuscript is accepted if required according to the journal that you are submitting your paper to) 


\section{Abstract}

In this work, we use the photoisomerization of azobenzenes, a phenanthrospirooxazine, and a fulgide in a twisted nematic liquid crystalline phase to change the chiral twisting power of the system. The changes are probed by the rotatory power of linearly polarized light. Time resolved and steady state experiments are carried out. The chiral change and the photoisomerization process have similar characteristic recovery times and activation energy, thus probing that the change is induced by the modification in the chemical composition of the photochromic dopant system. The amplitude of the light twisting power change correlates with the order change in the liquid crystal (LC) but not with the modification in the absorption characteristics of the system. This indicates that the driving force of the chiral change is the microscopic order modification in the LC phase that affects the helical pitch of the phase.

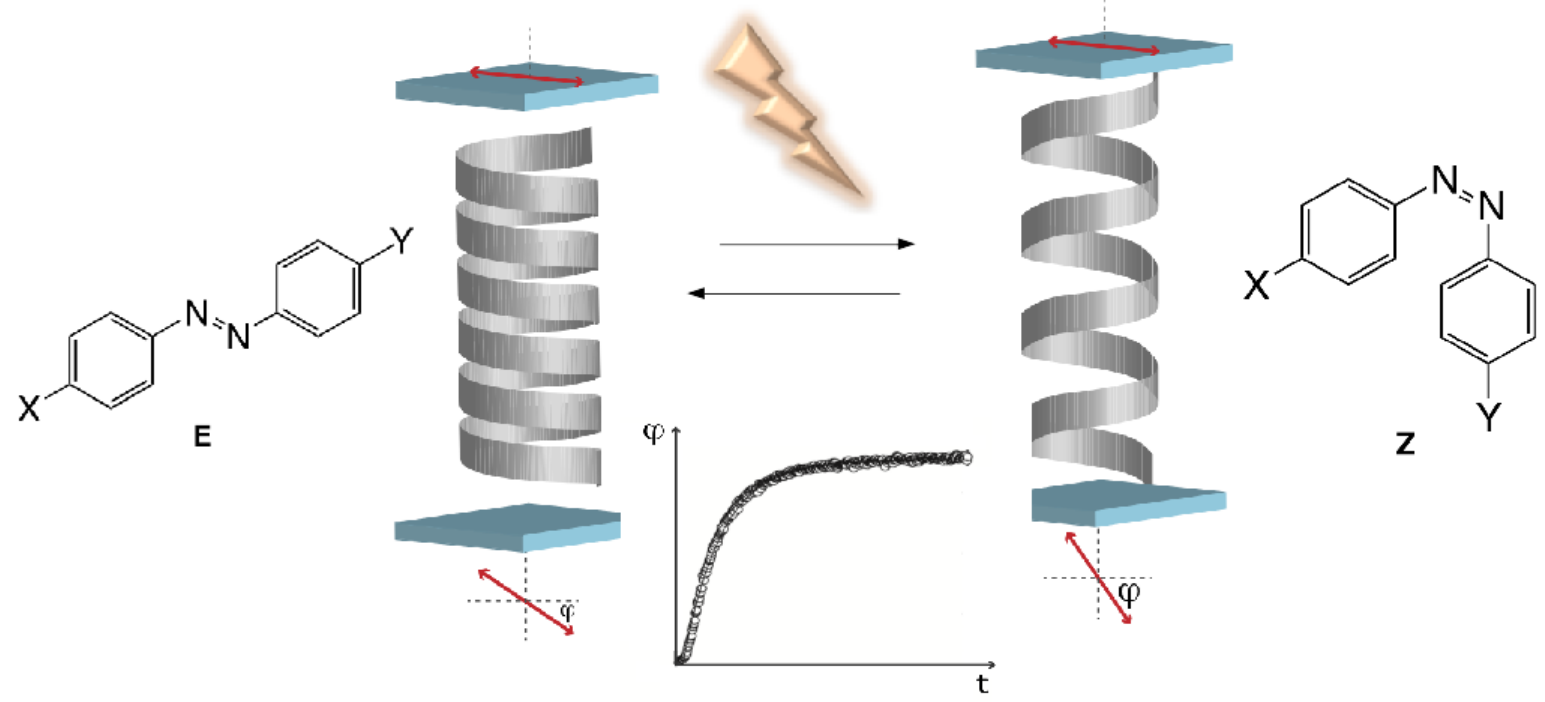

KEYWORDS: photoisomerization, liquid crystals, chiral power change, photochromism, azobenzenes, spirooxazine. 


\section{Introduction}

All-optical storage-read systems are interesting devices because of the possibility of wireless remote control. To achieve the operation of such a device, a photosensitive material must provide the state change of the system that can be tested by a suitable optical signal. In this regard, photochromic molecular systems are adequate to operate as the commanding element. They have been tested in many optical storage and photoswitching devices. ${ }^{1-4}$ In particular, a photochromic system, lacking thermal interconversion reactions, could provide a long-term memory, that can be read and erased with light. However, very few systems show this feature: namely some diarylethenes and fulgides. ${ }^{5}$ In all cases, a writing and an erasing wavelength has to be used in the suited range for the transformation in each direction to occur. On the other hand, systems with one photochemical conversion pathway and thermal reversion to the original state are also attractive because they offer the possibility of periodic excitation with a frequency limited by the inverse of the decay lifetime of the metastable state. In this perspective, there is no need of an erasing wavelength, whereas the readout must be performed during the lifetime of the metastable state. These requirements are fulfilled by a vast variety of systems, particularly azobenzenes and related compounds ${ }^{6-10}$ that display a great light stability and allow many excitation cycles. Nonetheless, readout is also a concern in these systems. Signals based on absorption of the metastable isomer can cause destructive reading (reversion of the signal) as readout and erasing wavelength ranges coincide, with the difference being that readout is performed at a much lower incident light power. Hereof, the use of light signals that are not based on absorption is an interesting alternative because they only cause small perturbations in the device, especially if the photochemically sensitive element is not involved. 
Liquid crystals (LC) are ideal media as hosts for photochromic systems. LC combine anisotropic properties of the solid state with the fluidity and solvation capability of the liquid state. The twisted nematic cell is the key element in LC displays. There are many reports on the use of LC doped with photochromic molecules. ${ }^{8,11-16}$ In this approach, the light transformation of the photochrome is used to induce changes in the LC environment that are amplified by the susceptibility of the metastable phase. The optical features of the liquid crystals were used to test the state of the system in most of the cases by the change in the reflection ${ }^{13-15}$ signal of twisted nematic phases. This testing does not involve absorption of the photochromic element. Another way of testing that does not imply destructive reading is based on refractive index changes, particularly in the optical rotatory dispersion in chiral phases, which can be monitored by the rotation of the plane of linearly polarized light. This specific approach avoids the need of spectral detection or of a different wavelength of the probe light for every system. For this reason optical probing of the state of the system based on refractive index changes is an interesting possibility.

Photochromic transformation is accompanied by a change in the affinity of the photoproduced isomer and the mesophase, which modifies the state of the labile LC. The particular aim of this work is the study of changes in the chiral power of a twisted nematic LC induced by the photoisomerization of photochromic compounds. Accordingly, we used three types of photochromic compounds. Their structures are shown in Scheme 1. Azobenzenes, having an E-Z phototransformation with a thermally metastable $\mathrm{Z}$ isomer. The geometry change upon this isomerization greatly perturbs the LC phase. A spirooxazine with a great shape and spectral absorption range change upon photoisomerization was the second family. Finally, we used a fulgide with absence of back thermal isomerization at room temperature and a very small shape change. 
<smiles>[X]c1ccc(/N=N/c2ccc(/N=N/c3ccc([X])cc3)cc2)cc1</smiles>

Azobenzene<smiles>C=C1c2ccccc2C2c3ccccc3C(=O)C(=NC=C3N(C)c4ccccc4C3(C)C)C12</smiles>

Spirooxazine
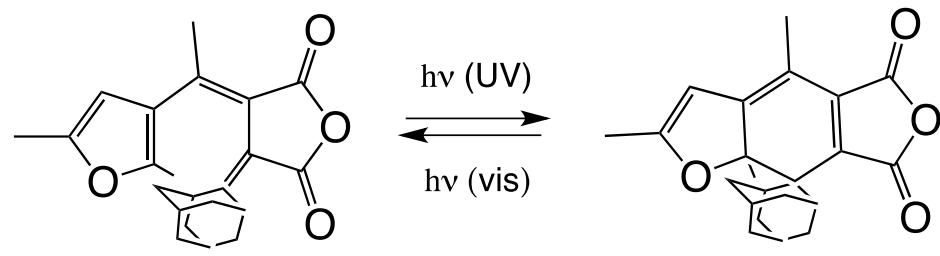

Fulgide

Scheme 1. Molecular structure of the photochromic compounds used in this work and their photochromic reactions.

\section{Material and Methods}

\subsection{Materials}

The following chemicals were used as received: Azobenzene (AB) (Aldrich), 4-(N,Ndimethylamino)azobenzene (DAB) (Aldrich), 2,3-dihydro-1,3,3-trimethylspiro[indoline-2,2'$[2 \mathrm{H}]$ phenanthro[9,10b][1,4]oxazine] (SO) (Aldrich), E- $\alpha$-(2,5-dimethyl-3-furyl ethylidene)(adamantylidene)-succinic anhydride (AFU) (Chemos $\mathrm{GmbH}$ ), commercial liquid crystal mixtures ZLI-1132 (Licristal/Merck) and S-811 chiral inductor (CI), compatible with ZLI-1132 phase and with a helical twisting power (HTP) of -13,9 $\mu \mathrm{m}^{-1} \mathrm{M}^{-1}$, was also from Licristal/Merck. 
4-(N,N-dimethylamino)-4' ${ }^{\prime}$-nitroazobenzene (N-DAB) was synthesized according to literature. $^{17}$ Cells of parallel polyimide rubbed layers, coated with indium tin oxide (ITO), spaced $10 \mu \mathrm{m}$ apart were purchased from E.H.C. Co., Ltd. (Japan).

\subsection{Sample Preparation}

Mixtures of the twisted nematic phase were prepared by weighting ZLI1132, S-811, the photochromic dye and then stirring for at least $6 \mathrm{~h}$ at $\sim 40-50{ }^{\circ} \mathrm{C}$ in the dark. Typically, the amount of the chiral inductor was adjusted between 5 and $15 \% \mathrm{w} / \mathrm{w}$ to tune the cholesteric reflection band in the NIR far from the absorption bands of the dyes and from the probe laser wavelength. Cells were filled at room temperature by capillar effect, and orientation was verified by polarized microscopy.

\subsection{Methods}

2.3.1. Absorption. A Shimadzu UV3101PC spectrophotometer was used for the absorption measurements. When necessary, the sample was irradiated immediately before the measurement.

2.3.2 Polarized light microscopy. Phase transition temperatures were recorded by observation of the disappearance and appearance of the typical cholesteric phase texture, when heating and cooling the samples, respectively. A transmission microscope (Leitz DMR) equipped with a heating stage (Leitz 1350) was used. The sample was placed between two crossed film polarizers (Melles Griot). The temperature, for which the sample starts showing isotropic phase, is determined by the appearance of the the first black point in the sample. The disappearance of the last bright spot determines the end of the phase transition temperature range, $\mathrm{T}_{\mathrm{CH}-\mathrm{I}}$. Determination of the $\mathrm{T}_{\mathrm{CH}-\mathrm{I}}$, was performed in quadruplicate and the results were comparable within experimental uncertainty. 
2.3.3 Chiral Power. We setup an optical arrangement to measure the intensity of linearly polarized light transmitted through the sample as a function of time and angle of the analyzer. Briefly, the sample was placed in a thermostatic cell holder between two Glan-Thompson polarizers (Melles Griot) and laser light of $785 \mathrm{~nm}$ (Melles Griot) transmitted by the system was measured. The optical axis of the sample front window is aligned in the vertical position $\left(0^{\circ}\right)$. The position of the polarizer is placed at $0^{\circ}$ in all measurements, while the orientation of the analyzer is varied between 0 and $120^{\circ}$. The intensity of the analysis light was controlled with a variable neutral density filter, to avoid detector saturation. To ensure reproducibility of the measurements, the photolyzing xenon flash lamp was fixed to the cell holder. The temperature was recorded with a platinum resistance. To detect the transmitted light through the sample, a silicon diode (Silicon Detector Corporation) was used. To minimize spurious light from the photolysis source and ambient light, a cut-off filter (RG715, Schott) was placed in front of the diode. The time-dependent signal was recorded for different orientations of the analyzer, with an oscilloscope HP54502A (Hewlett Packard, $400 \mathrm{MHz}$ ), and stored in a PC.

\section{Results}

Observation of the samples in the microscope, sandwiched between polarizers, allowed the verification of the persistence of cholesteric phase after addition of the photochromic dopant, as well as to determine the phase transition temperature range from the chiral to the isotropic

phase, $\mathrm{T}_{\mathrm{CH}-\mathrm{I}}$. Table 1 presents the phase transition temperature range for all of the studied samples. 
Table 1. Transition Phase Temperature Range, $\mathrm{T}_{\mathrm{CH}-\mathrm{I}}$, from the Cholesteric to Isotropic Phase for all the studied samples.

\begin{tabular}{c|c|c|c} 
& $\begin{array}{c}\text { Probe } \\
(\boldsymbol{\%} \mathbf{w} / \mathbf{w})\end{array}$ & $\begin{array}{c}\mathbf{S - 8 1 1} \\
(\boldsymbol{\%} \mathbf{w} / \mathbf{w})\end{array}$ & $\begin{array}{c}\mathbf{T}_{\mathbf{C H}-\mathbf{I}} \\
\left({ }^{\circ} \mathbf{C}\right)\end{array}$ \\
\hline $\mathbf{D}$ DAB & 5.0 & 10.0 & $(59-62) \pm 2$ \\
& 0.5 & 5.5 & $(63-71) \pm 2$ \\
\hline \multirow{2}{*}{ N-DAB } & 0.6 & 11.6 & $(59-65) \pm 2$ \\
& 0.4 & 15.0 & $(53-60) \pm 2$ \\
\hline Fulgide & 4.6 & 10.0 & $(58-63) \pm 2$
\end{tabular}

Samples with the same concentration of CI $(\sim 10 \% \mathrm{w} / \mathrm{w})$ present similar $\mathrm{T}_{\mathrm{CH}-\mathrm{I}}$. As expected, increasing $\mathrm{CI}$ concentration, leads to decrease in the $\mathrm{T}_{\mathrm{CH}-\mathrm{I}}$. To ensure that the cholesteric order was maintained throughout the experiment, chiral power experiments were carried out at temperatures sufficiently below $\mathrm{T}_{\mathrm{CH}-\mathrm{I}}$.

The position of the cholesteric band was regulated in order to prevent interfering with the absorption of the photochromic system. Figure 1, shows the transmission spectra for different samples of N-DAB and SO, prepared with different concentrations of CI. The band at $500 \mathrm{~nm}$ corresponds to the absorption of the E isomer of N-DAB whereas the bands at 1100 and $2200 \mathrm{~nm}$ correspond to the cholesteric reflection band when the $\mathrm{CI}$ is 11.0 and $5.5 \% \mathrm{w} / \mathrm{w}$, respectively. For SO samples, the cholesteric band is at $1400 \mathrm{~nm}$, while the merocyanine absorption is seen at 600 $\mathrm{nm}$. 


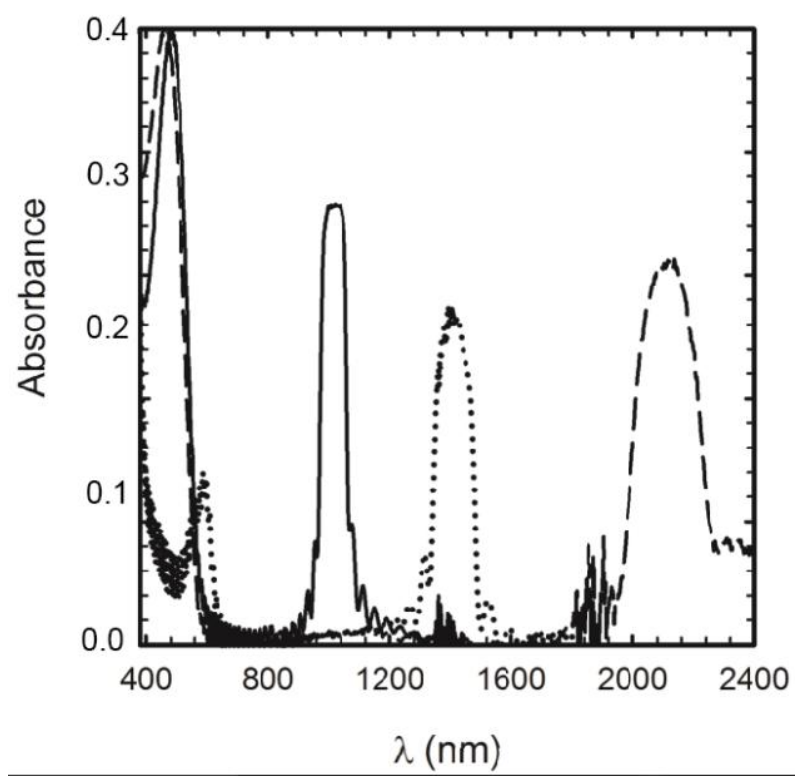

Figure 1. Absorption spectra (10 Om cell thickness) of samples of $0.5 \% \mathrm{w} / \mathrm{w}$ N-DAB in ZLI with 5.5\% w/w S-811 (dashed line), 0.6\% w/w N-DAB in ZLI with 11.0\% w/w S-811 (full line), and $4.6 \% \mathrm{w} / \mathrm{w}$ SO in ZLI with 10.0\% w/w S-811 (dotted line).

\subsection{Chiral Power Change}

The transient of the transmitted light at different analyzer orientations $\left(\theta_{a n}\right)$, between 0 and $120^{\circ}$, was monitored after pulsed excitation of the photochromic component. Photoisomerization of this component induces perturbations in the LC phase that changes the refractive index for right and left handed circularly polarized light, thus resulting in a rotation of the angle of the linearly polarized light passing through the sample. The angle and time dependent intensity transmitted by the sample, can be represented by a matrix $\left(\left[\mathrm{I}\left(\theta_{a n}, \mathrm{t}\right)\right]\right)$, recorded for each fixed temperature. Its analysis allows the reconstruction of the polarization state of the light, obtaining a Malus curve, at any time after and before the photolyzing flash. Figure 2 shows typical transients of $\mathrm{I}\left(\theta_{a n}, \mathrm{t}\right)$ for two different analyzer orientations for a sample of $5.0 \% \mathrm{w} / \mathrm{w}$ DAB $(10.0 \% \mathrm{w} / \mathrm{w}$ S-811 in ZLI-1132). 


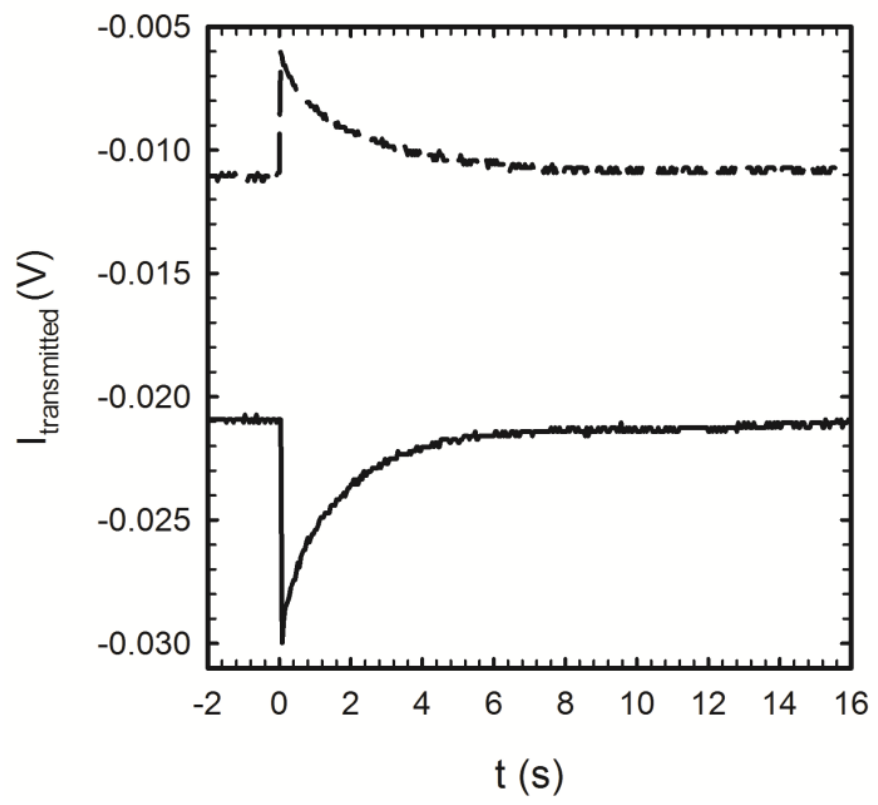

Figure 2. Typical decay curves of light transmitted through the sample, $I\left(\theta_{a n}, t\right)$, after a flash for two different positions of the analyzer $\left(90^{\circ}\right.$, dashed line, and $70^{\circ}$, solid line) for a sample of $5.0 \% \mathrm{w} / \mathrm{w}$ DAB in a $10.0 \% \mathrm{w} / \mathrm{w}$ S-811 in ZLI-1132 mixture, contained in a 10 Om cell.

From the phase shift parameter values, $\varphi$, obtained by fitting the $\left[\mathrm{I}\left(\theta_{a n}, \mathrm{t}\right)\right]$ data to Eq. 1 , at each fixed time after the photoconversion, the temporal variation of the optical phase delay can be adjusted assuming first order kinetics for the thermal recovery of the probe, Eq. 2.

$$
\begin{gathered}
\Delta J\left(\theta_{a n}, t\right)=I\left(\theta_{a n}, t\right)-I\left(\theta_{a n}, t<0\right)=A \cdot \cos ^{2}\left(\theta_{a n}+\varphi(t)\right)+C \\
\varphi(t)=\Delta \phi \cdot e^{-k t}-\phi_{i}
\end{gathered}
$$

In Figure 3, the modulation, $A$, and residual, $C$, amplitude parameters of the polarized light transmitted through the sample are shown, as a function of time. It can be seen that the state of polarization is always linear, since the maximum transmitted light intensity is constant to within $0.1 \%$ and its minimum is zero also to within $0.1 \%$ of the maximum. The small perturbation in the 
total intensity (Figure 3, left) may be due to slight changes in the reflection at the glass LC interphase because of small refractive index changes.
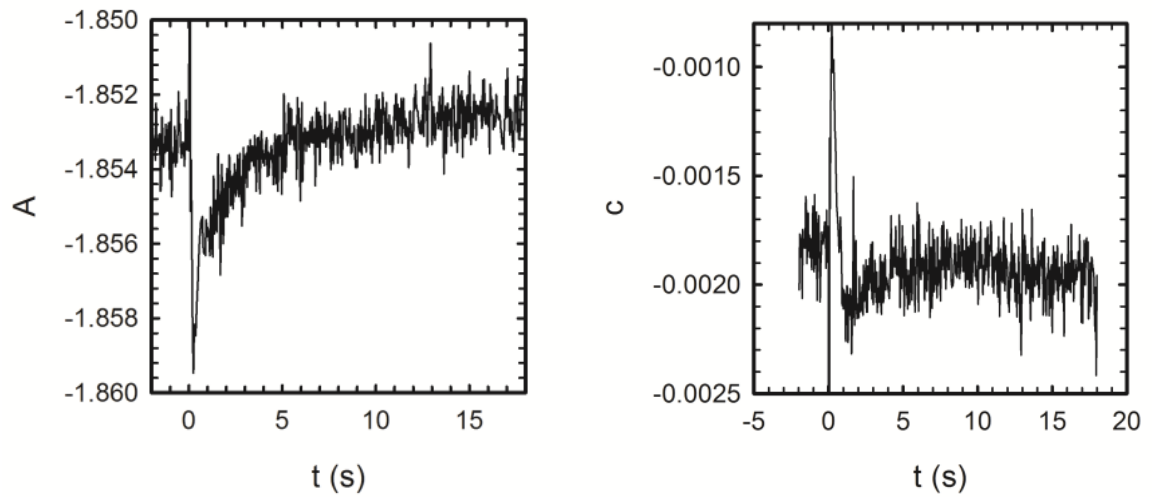

Figure 3. Modulation (left; A from Eq. 1) and residual (right, C from Eq. 1) amplitude parameters of the polarized light transmitted through the sample as a function of time for a sample of $5.0 \% \mathrm{w} / \mathrm{w}$ DAB in a $10.0 \% \mathrm{w} / \mathrm{w} \mathrm{S}-811$ in ZLI-1132 mixture, contained in a $10 \mathrm{Om}$ cell.

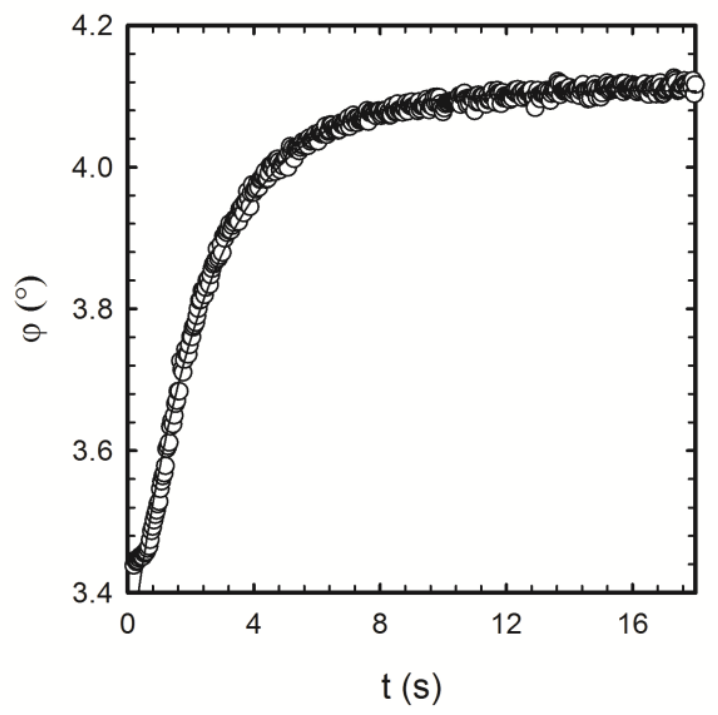

Figure 4. Phase delay decay as a function of time for a sample of $5.0 \% \mathrm{w} / \mathrm{w}$ DAB in a $10.0 \%$ $\mathrm{w} / \mathrm{w}$ S-811 in ZLI-1132 mixture, thermostatized at $57^{\circ} \mathrm{C}$. The line represents the 
monoexponential fit of the decay of the phase delay: $\varphi(t)=-0.015 \mathrm{rad} \cdot e\left(-0.459 \mathrm{~s}^{-1} \cdot t\right)+0.072 \mathrm{rad}$.

The rate constant, $k$, for the isomerization reaction is obtained from the fitted values of the phase delay as a function of time. The phase delay is manifested as a twist in the plane of polarization of the linearly polarized light transmitted by the sample. It arises on the change in helical twisting power of the medium caused by the change in refractive index, photoinduced by the isomerization. In all cases, azobenzene or spirooxazine as dopants, the thermal recovery kinetics of the initial state has a monoexponetial behavior.

The activation energy $\left(E_{\mathrm{a}}\right)$, preexponential factor $(A)$, and activation entropy $\left(\Delta S^{\ddagger}\right)$ (reference state, $\mathrm{C}_{0}=1 \mathrm{M}$ ), of the photoisomerization rate constant in the LC phase are calculated from parameters of the Arrhenius plot (Eq. 3 and 4).

$$
\begin{aligned}
& \ln k=\ln A- \frac{E_{a}}{R T} \\
& \Delta S^{\neq}=R \cdot \ln \left(\frac{A \cdot h}{k_{B} \cdot T \cdot e}\right)
\end{aligned}
$$

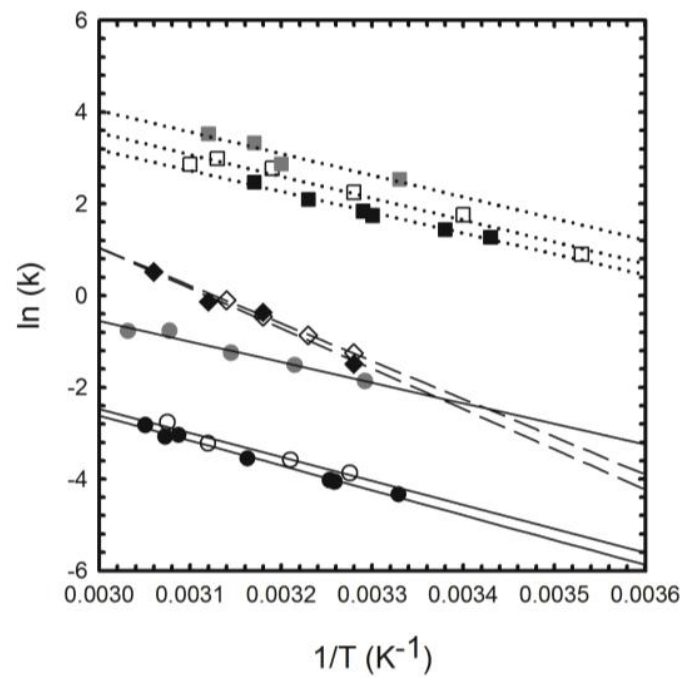


Figure 5. Arrhenius plots for the decay rate constant of the chiral twisting power for different photochromes in twisted nematic ZLI-1132 + S-811: 5.0\% w/w DAB, $10.0 \% \mathrm{w} / \mathrm{w}$ S-811 (squares); 4.6\% w/w SO, 9.8\% w/w S-811 (diamonds); and for samples of $0.5 \% \mathrm{w} / \mathrm{w} \mathrm{N}-\mathrm{DAB}$ with $5.0 \% \mathrm{w} / \mathrm{w}$ (full circles), $11.0 \% \mathrm{w} / \mathrm{w}$ (empty circles) and 15.0\% w/w (grey circles) S-811. Independently prepared samples of $\mathrm{DAB}$ and $\mathrm{SO}$ with the same probe and $\mathrm{CI}$ concentrations as depicted previously are represented by the different filled symbols. The lines represent the linear fits to Eq. 3.

The Arrhenius plots, in Figure 5, show that for the same probe in the cholesteric media, all the lines have the same slope within experimental error. Nevertheless, for single samples of the same dopant, or for samples with different CI concentration of the same probe, the value of the intercept differs significantly. This is related to the local order of the system, as discussed below. This was already observed for the isomerization of these same photochromes in nematic or cholesteric phases when measuring differences in absorption of the isomers. ${ }^{17,18}$

The activation energy does not change from experience to experience, within experimental error, however the activation entropy does. The activation entropy reflects the changes in the order of probe and environment in a balanced system of metastable isomer and transition state. For azobenzenes and $\mathrm{SO}$, there is a great change in molecular shape between the metastable isomer and the transition state. Isomerization reactions of azobenzenes and SO in ordered phases have a great entropic control with a large component provided by the variation of microscopic order of the medium. Changes in the activation entropy can be explained, if one admits that the microscopic order of the phase around the dopand is highly variable between samples. The overall order of the macroscopic system depends on the details of the cell construction (quality of the orientation, thickness variations) that can slightly affect the pitch. 
Macroscopic order probably affects the microscopic order causing changes in the entropy of activation.

Table 2 summarizes the results obtained for the Arrhenius parameters of the first order rate constant of the thermal recovery for azobenzenes and $\mathrm{SO}$ in the twisted nematic phases, compared to the values in common solvents from literature. The activation energies and the characteristic times of the photoisomerization processes and of the chiral changes are similar, indicating that change is induced by the modification in the chemical structure of the dopants.

Table 2. Comparison of the Arrhenius Parameters for the Thermal Decay of the Photoisomer and of the Chiral Changes of the Photochromic Systems after Light Pulse Irradiation. References: a) Bossi et al. ${ }^{17}$; b)Nishimura et al. ${ }^{19}$ c) Asano et al. ${ }^{20}$ d) Favaro et al. ${ }^{21}$

\begin{tabular}{ccccccc}
\hline & \multicolumn{2}{c}{$\begin{array}{c}\text { Twisted Nematic } \\
(\mathbf{c h i r a l ~ p o w e r})\end{array}$} & \multicolumn{2}{c}{ Twisted Nematic } & \multicolumn{2}{c}{$\begin{array}{c}\text { Solvents } \\
(\text { isomer lifetime })\end{array}$} \\
\cline { 2 - 7 } & $\boldsymbol{E}_{\mathbf{a}}$ & $\Delta \boldsymbol{S}^{\ddagger}$ & $\boldsymbol{E}_{\mathrm{a}}$ & $\Delta \boldsymbol{S}^{\ddagger}$ & $\boldsymbol{E}_{\mathbf{a}}$ & $\Delta \boldsymbol{S}^{\ddagger}$ \\
$(\mathbf{k J} / \mathbf{m o l})$ & $(\mathbf{J} /(\mathbf{m o l} \mathbf{K}))$ & $(\mathbf{k J} / \mathbf{m o l})$ & $(\mathbf{J} /(\mathbf{m o l ~ K}))$ & $(\mathbf{k J} / \mathbf{m o l})$ & $(\mathbf{J} /(\mathbf{m o l ~ K}))$ \\
\hline DAB & $45 \pm 2$ & $-146 \pm 6$ & $52 \pm 3$ & $-100 \pm 7$ & $88^{\mathrm{b}}$ & $-11^{\mathrm{b}}$ \\
N-DAB & $38 \pm 2$ & $-107 \pm 16$ & $46 \pm 1$ & $-87 \pm 2$ & $45-64^{\mathrm{c}}$ & $-16 /-31^{\mathrm{c}}$ \\
SO & $70 \pm 2$ & $-38 \pm 7$ & $82 \pm 2$ & $-3 \pm 1$ & $66-72^{\mathrm{d}}$ & $-47 /-23^{\mathrm{d}}$
\end{tabular}

The effect of photoisomerization on the amplitude of the chiral change of the sample was studied also as a function of temperature and of the concentrations of CI. Figure 6 shows that the amplitude of the chiral change shows no clear tendency neither with temperature nor with the CI concentration. 


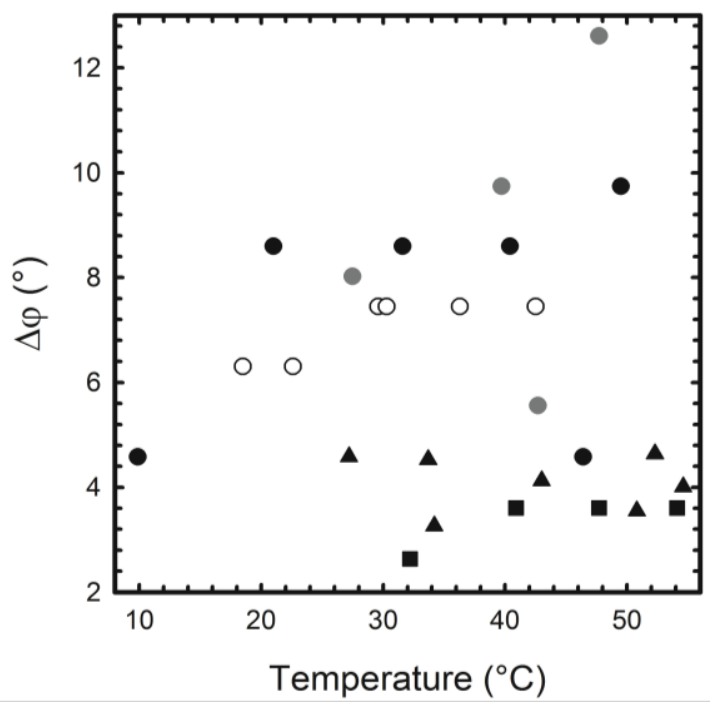

Figure 6. Phase shift amplitude for the rotation of linearly polarized light as a function of temperature for different photochromes in twisted nematic ZLI-1132 + S-811: 5.0\% w/w DAB, $10.0 \% \mathrm{w} / \mathrm{w} \mathrm{S}-811$ (squares), $4.6 \% \mathrm{SO}, 9.8 \% \mathrm{w} / \mathrm{w} \mathrm{S}-811$ (triangles), and for samples of 0.5\% w/w $\mathrm{N}-\mathrm{DAB}$ with $5.0 \% \mathrm{w} / \mathrm{w}$ (full circles), $11.0 \% \mathrm{w} / \mathrm{w}$ (empty circles) and 15.0\% w/w (grey circles) S-811. The amplitude of the data for DAB (squares) was divided by 10 in order to fit in the graph scale. All samples were contained in 10 Om cells.

Phase shift was obtained for a sample of azobenzene $(\mathrm{AB})(9.0 \% \mathrm{w} / \mathrm{w})$ in cholesteric phase $(\mathrm{S}-81110 \% \mathrm{w} / \mathrm{w})$ as a function of the conversion to the $\mathrm{Z}$ isomer. This was achieved by successive flash light irradiation at a frequency much higher than the decay rate constant. The lifetime of the $\mathrm{Z}$ isomer of $\mathrm{AB}$ is in the order of several hours. ${ }^{22}$ This allows recording the light transmitted by the analyzer in all orientations $\left(0^{\circ}\right.$ to $\left.360^{\circ}\right)$ after irradiation, without causing an appreciable decay of the $\mathrm{Z}$ isomer during the measurement. The transmitted signal was averaged between 2 to 3 seconds.

Figure 7 shows the phase shift, measured relative to the unirradiated sample, as a function of the irradiation light dose, represented by the number of flashes. Experimental data were fitted 
with an empirical exponential growth function, and the following relation: $\Delta \varphi=\varphi(N)-\varphi(N=0)=0.063 \mathrm{rad} \cdot\left(1-e^{-0.030 N}\right)$ was found, where $\mathrm{N}$ is the number of flashes.

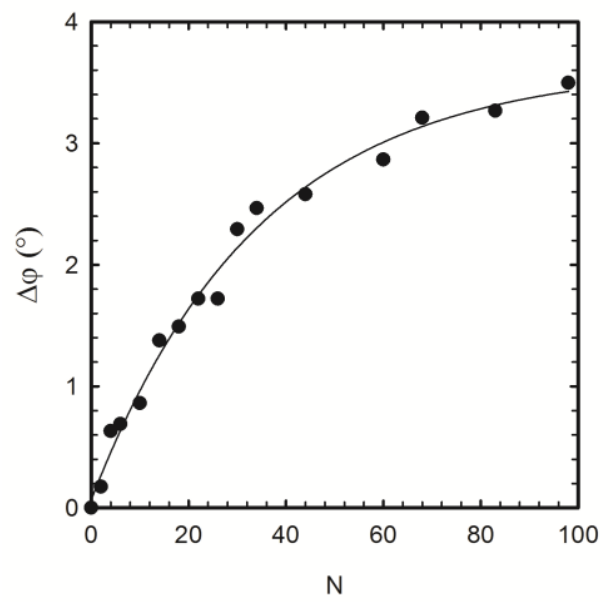

Figure 7. Phase shift amplitude, $\Delta \varphi$, Eq. 1., relative to the unirradiated sample, as function of the number of flashes for a sample of $8.0 \% \mathrm{w} / \mathrm{w} \mathrm{AB}$ in a $9.0 \% \mathrm{w} / \mathrm{w} \mathrm{S}-811$ in ZLI-1132 mixture, contained in a $10 \mathrm{Om}$ cell. The line represents the monoexponential fit to the equation in the text.

Figure 7 represents a conversion curve from the thermodinamically stable $\mathrm{E}$ isomer of $\mathrm{AB}$ to the photostationary E-Z mixture, and demonstrates a correlation between the twist angle of the polarization plane of the transmitted light and the photoconversion of the photochromic system. As the number of flashes increases, the variation of the phase shift grows in magnitude until it reaches saturation at the photostationary state: representing the maximum achievable conversion to the $\mathrm{Z}$ photoisomer. These experiments illustrate the arrival of the system to a photostationary state, the additivity of the light stimuli, and the reproducibility of the photolyzing light pulses.

For a sample with AFU 2.1\% w/w (10.0\% w/w S-811), two sources of light were used alternatively for the conversion of the probe; a LED of $365 \mathrm{~nm}$ and an $\mathrm{Hg}$ arc lamp with a band pass filter (GG 455), that fundamentally emits light of $546 \mathrm{~nm}$. To ensure that the conversion of 
one form to another is complete, previous experiences measuring the change in the system through absorption spectra (Figure 8) were performed and the irradiation time (about $15 \mathrm{~min}$ ) determined. The isomerization of AFU does not cause any change in the helical pitch of the twisted nematic phase, as no change in the orientation of the linearly polarized light could be measured. AFU is a system that presents a large spectral change near the wavelength of analysis but its photoisomerization, presents no variation in the pitch of the chiral phase, due to small changes of molecular shape between the two isomers.

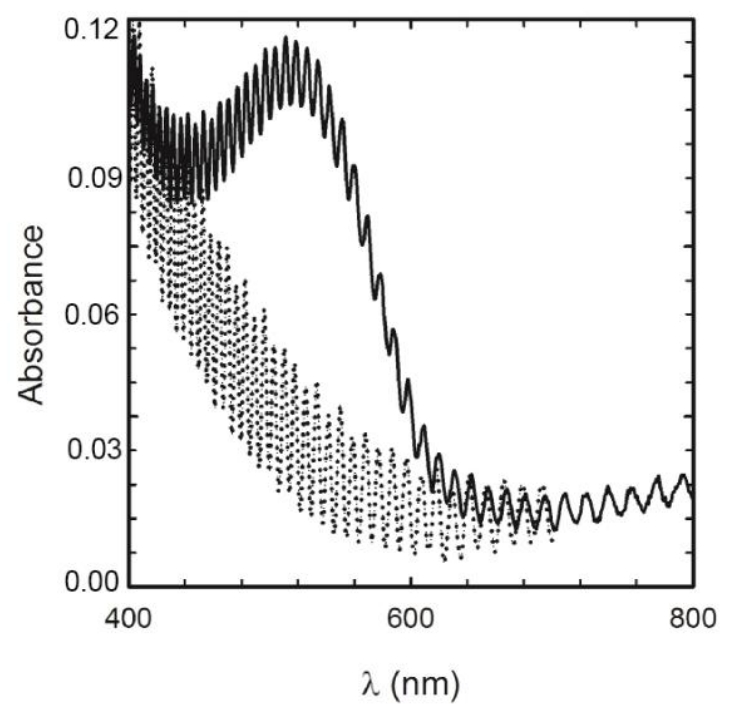

Figure 8. Absorption spectra of the open (full line) and closed (dashed line) form of AFU, for a sample of $2.1 \% \mathrm{w} / \mathrm{w}$ AFU in ZLI with $10.0 \% \mathrm{w} / \mathrm{w}$ S-811, contained in a $10 \mathrm{Om}$ cell.

\section{Discussion}

The traditional way of measuring the capacity of rotation of the polarization plane of light is through the quantity called optical activity, $[\alpha]_{\lambda}^{T}$, defined as the specific rotation per unit length of traveled light and per unit concentration (see Eq. 5). Photochromic compound concentration in molarity, is obtained by approximating the density of the medium to $1 \mathrm{~g} / \mathrm{cm}^{3}$ and 
by assuming complete conversion to the photoisomer in the photostationary state. The thickness of the cells was $10 \mu \mathrm{m}$ in all cases.

$$
\frac{[\alpha]_{\lambda}^{T}}{c m \cdot M}=\frac{\alpha}{l . c}=\frac{\Delta \varphi\left(^{o}\right) \cdot 10^{3}}{l(c m) \cdot c_{\text {probe }}(M)}
$$

The specific rotation, obtained for samples with $0.5 \% \mathrm{w} / \mathrm{w}$ of N-DAB is around $5 \times 10^{3}$ degrees $(\mathrm{cm} \cdot \mathrm{M})^{-1}$, while for a glucose solution in water it is of the order of $1 \times 10^{2}$ degrees $(\mathrm{cm} \cdot \mathrm{M})^{-1}$. Upon photoisomerization, azobenzenes generate a great alteration in the chiral environment that can be understood by the the high difference of affinity between the two isomers and the mesophase. The chiral phase amplifies the alterations produced by the probe. The changes in transmitted light induced by the photoisomerization of the studied compounds do not involve absorption of light, since the light is always linearly polarized, it keeps the same amplitude, and birefringence is not observed. The thermal photoisomerization of the probe is instantaneously accompanied by the reordering of the environment because this change of order occurs in the milliseconds timescale. For this reason, the decay lifetimes of the chiral changes and its observed activation parameters are controlled by the isomerization process. The kinetic parameters obtained in LC differ from the typical values reported in solution, see Table 2, being DAB the probe that shows the highest changes: both, $E_{\mathrm{a}}$ and $\Delta S^{\neq}$are smaller to the ones reported by literature in solution. This can be understood, as the $\mathrm{Z}$ isomer has low affinity for the chiral nematic phase, while the in plane transition state, can be better ordered in the LC media. Further, the transition state for the $\mathrm{Z} \rightarrow \mathrm{E}$ transformation has a linear geometry, so that it is more stabilized in the LC than the bent $\mathrm{Z}$ isomer, from the energetic point of view, while the entropy of the ensamble decreases compared to the $\mathrm{Z}$ state. These facts justify the lower activation energy 
with respect to the one in solution, while they reflect in the large negative value of the activation entropy.

By contrast, the SO presents an activation energy and entropy comparable to those obtained in solution. The smaller entropy value (in modulus) for SO with respect of the azobenzenes can be explained as the merocyanine (open isomer), with an extended geometry can stabilize better the chiral phase, whereas the more globular-like spiro form of the transition state is destabilized. Phenanthrospirooxazine (SO) and azobenzenes induce appreciable changes in chiral twisting power with an adequate signal to noise ratio. Absorption changes in these systems upon photoisomerization are high for SO, while azobenzenes show moderate spectral changes that are also more distant to the wavelength of analysis. On the other hand, AFU does not change the chiral twisting power of the phase upon isomerization. The fulgides show the highest absorption change and the lowest shape change upon photoisomerization of the three dopant types. Chiral changes can in principle be induced in two ways: 1) by means of variations in the absorption spectra, which generate a change in the circular dichroism, or 2) by changes in the helical pitch of the phase, caused by microscopic order modification in the LC. The amplitude of the light twisting power change correlates with the order change in the LC but not with the modification in the absorption characteristics of the system. These points out, that the driving force of the chiral change is originated in the change in the helical pitch of the phase.

\section{Conclusions}

In this work, the effect of the isomerization of the probes on the chiral nematic phase could be quantified by measurements of the phase shift in the polarization direction of light transmitted by the aligned chiral phase. Phenanthrospirooxazine and azobenzenes induce 
changes in the rotation of the cholesteric phase with adequate signal to noise ratio, that correlate with the order change in the local environment of the probe upon isomerization. In contrast, fulgide photoisomerization, generates no appreciable changes on the chirality of the medium.

\section{Acknowledgments:}

SS performed her work with fellowships from Universidad de Buenos Aires and CONICET. PFA is research staff from CONICET. The work was performed under financial support from UBA (Grants X086 and 20020100100234) and CONICET (PIP 11220100100397).

\section{References:}

(1) Crano, J. C., and Guglielmetti, R. J. (1999) Organic Photochromic and Thermochromic Compounds, p 378. Plenum Press, New York.

(2) Dürr, H., and Bouas-Laurent, H. (2003) Photochromism. Molecules and Systems, p 1218. Elsevier Science, Amsterdam.

(3) Bossi, M. L., and Aramendía, P. F. (2011) Photomodulation of macroscopic properties. $J$. Photochem. Photobiol. C Photochem. Rev. 12, 154-166.

(4) (2000) Photochromism. Molecules and switches. Thematic issue. Chem. Rev. 100, 6-1890.

(5) Kungwatchakun, D., and Irie, M. (1988) Photoresponsive polymers. Photocontrol of the phase separation temperature of aqueous solutions of poly-[N-isopropylacrylamide-co-N-(4phenylazophenyl)acrylamide]. Die Makromol. Chemie, Rapid Commun. 9, 243-246.

(6) Bandara, H. M. D., and Burdette, S. C. (2012) Photoisomerization in different classes of azobenzene. Chem. Soc. Rev. 41, 1809-1825.

(7) Garcia-Amorós, J., Gómez, E., Vallés, E., and Velasco, D. (2012) Photo-controllable electronic switches based on azopyridine derivatives. Chem. Commun. 48, 9080-9082.

(8) Tamaoki, N., and Kamei, T. (2010) Reversible photo-regulation of the properties of liquid crystals doped with photochromic compounds. J. Photochem. Photobiol. C Photochem. Rev. 11, $47-61$. 
(9) White, T. J., McConney, M. E., and Bunning, T. J. (2010) Dynamic color in stimuliresponsive cholesteric liquid crystals. J. Mater. Chem. 20, 9832-9847.

(10) Li, Y., and Li, Q. (2014) Nanoscience with liquid crystals: From self-organized nanostructures to applications, in Nanoscience with liquid crystals, From self-organized nanostructures to applications. NanoScience and Technology. (Li, Q., Ed.), pp 135-177. Springer International Publishing, Switzerland.

(11) Yamaguchi, T., Inagawa, T., Nakazumi, H., Irie, S., and Irie, M. (2001) Photoinduced pitch changes in chiral nematic liquid crystals formed by doping with chiral diarylethene. J. Mater. Chem. 11, 2453-2458.

(12) Ruslim, C., and Ichimura, K. (2002) Photoswitching in chiral nematic liquid crystals: interaction-selective helical twist inversion by a single chiral dopant. J. Mater. Chem. 12, 33773379.

(13) Lee, H.-K., Doi, K., Harada, H., Tsutsumi, O., Kanazawa, A., Shiono, T., and Ikeda, T. (2000) Photochemical Modulation of Color and Transmittance in Chiral Nematic Liquid Crystal Containing an Azobenzene as a Photosensitive Chromophore. J. Phys. Chem. B 104, 7023-7028.

(14) Li, Y., and Li, Q. (2012) Photochemically reversible and thermally stable axially chiral diarylethene switches. Org. Lett. 14, 4362-4365.

(15) Hrozhyk, U. A., Serak, S. V, Tabiryan, N. V, White, T. J., and Bunning, T. J. (2010) Optically switchable, rapidly relaxing cholesteric liquid crystal reflectors. Opt. Express 18, 9651-9657.

(16) Delden, R. A. van, Mecca, T., Rosini, C., and Feringa, B. L. (2004) A Chiroptical Molecular Switch with Distinct Chiral and Photochromic Entities and Its Application in Optical Switching of a Cholesteric Liquid Crystal. Chem. - A Eur. J. 10, 61-70.

(17) Bossi, M. L., Murgida, D. H., and Aramendía, P. F. (2006) Photoisomerization of Azobenzenes and Spirocompounds in Nematic and in Twisted Nematic Liquid Crystals. J. Phys. Chem. B 110, 13804-13811.

(18) Bossi, M. L., Rodríguez, J. B., and Aramendía, P. F. (2006) Photoinduced changes of absorption and circular dichroism in a chiral nematic phase containing a photochromic fulgide. $J$. Photochem. Photobiol. A Chem. 179, 35-41.

(19) Norio Nishimura, Toshinobu Sueyoshi, Hideyuki Yamanaka, Etsuko Imai, Shunzo Yamamoto, and S. H. (1976) Thermal Cis-to-Trans Isomerization of Substituted Azobenzenes II. Substituent and Solvent Effects. Bull. Chem. Soc. Jpn. 49, 1381-1387.

(20) Asano, T., Okada, T., Shinkai, S., Shigematsu, K., Kusano, Y., and Manabe, O. (1981) Temperature and pressure dependences of thermal cis-to-trans isomerization of azobenzenes which evidence an inversion mechanism. J. Am. Chem. Soc. 103, 5161-5165. 
(21) Favaro, G., Masetti, F., Mazzucato, U., Ottavi, G., Allegrini, P., and Malatesta, V. (1994) Photochromism, thermochromism and solvatochromism of some spiro[indolinoxazine]photomerocyanine systems: effects of structure and solvent. J. Chem. Soc. Faraday Trans. 90, 333-338.

(22) Hartley, G. S. (1938) The cis-form of azobenzene and the velocity of the thermal cis-transconversion of azobenzene and some derivatives. J. Chem. Soc. 113, 633-642. 


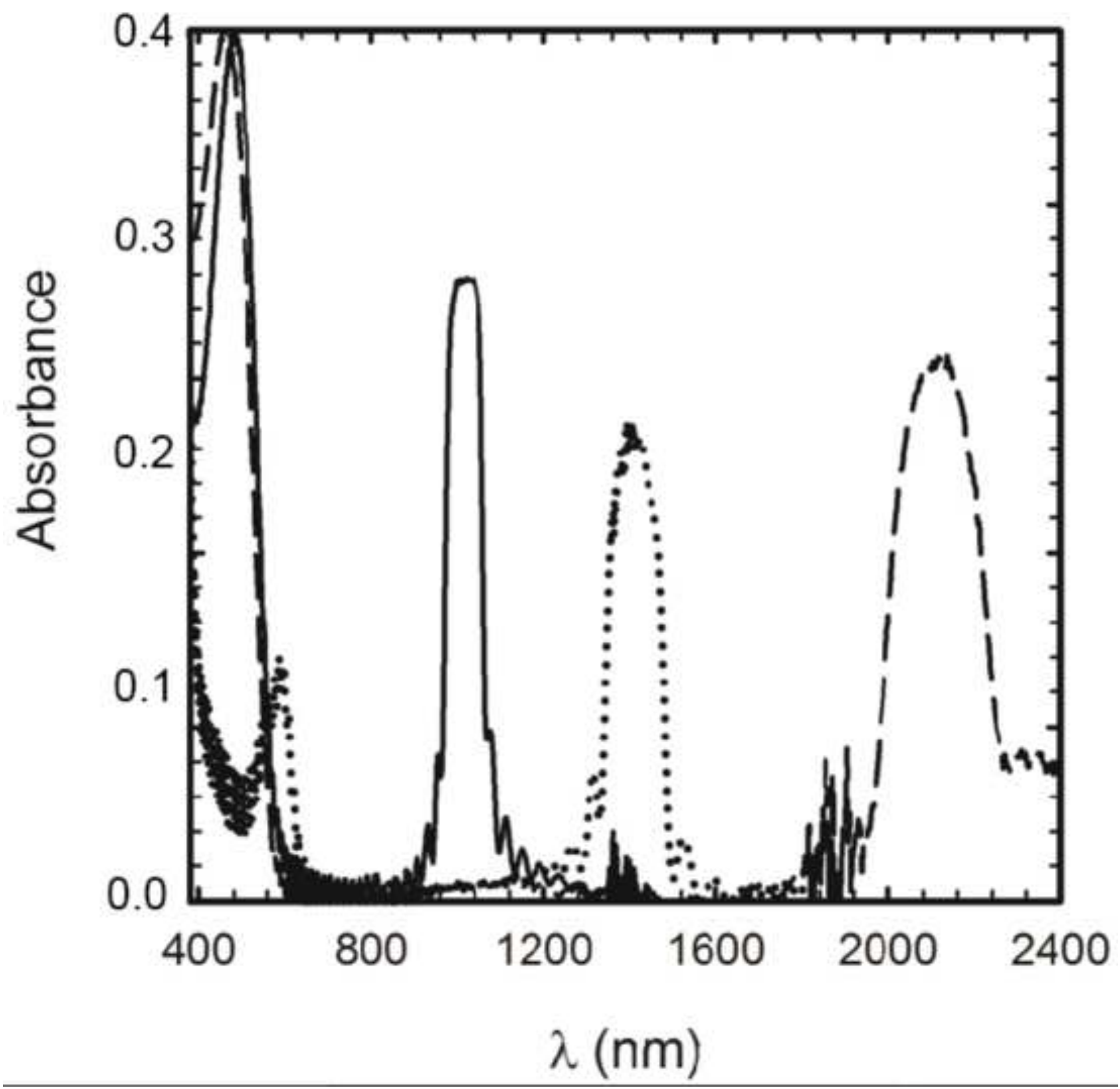


Figure8
Click here to download high resolution image

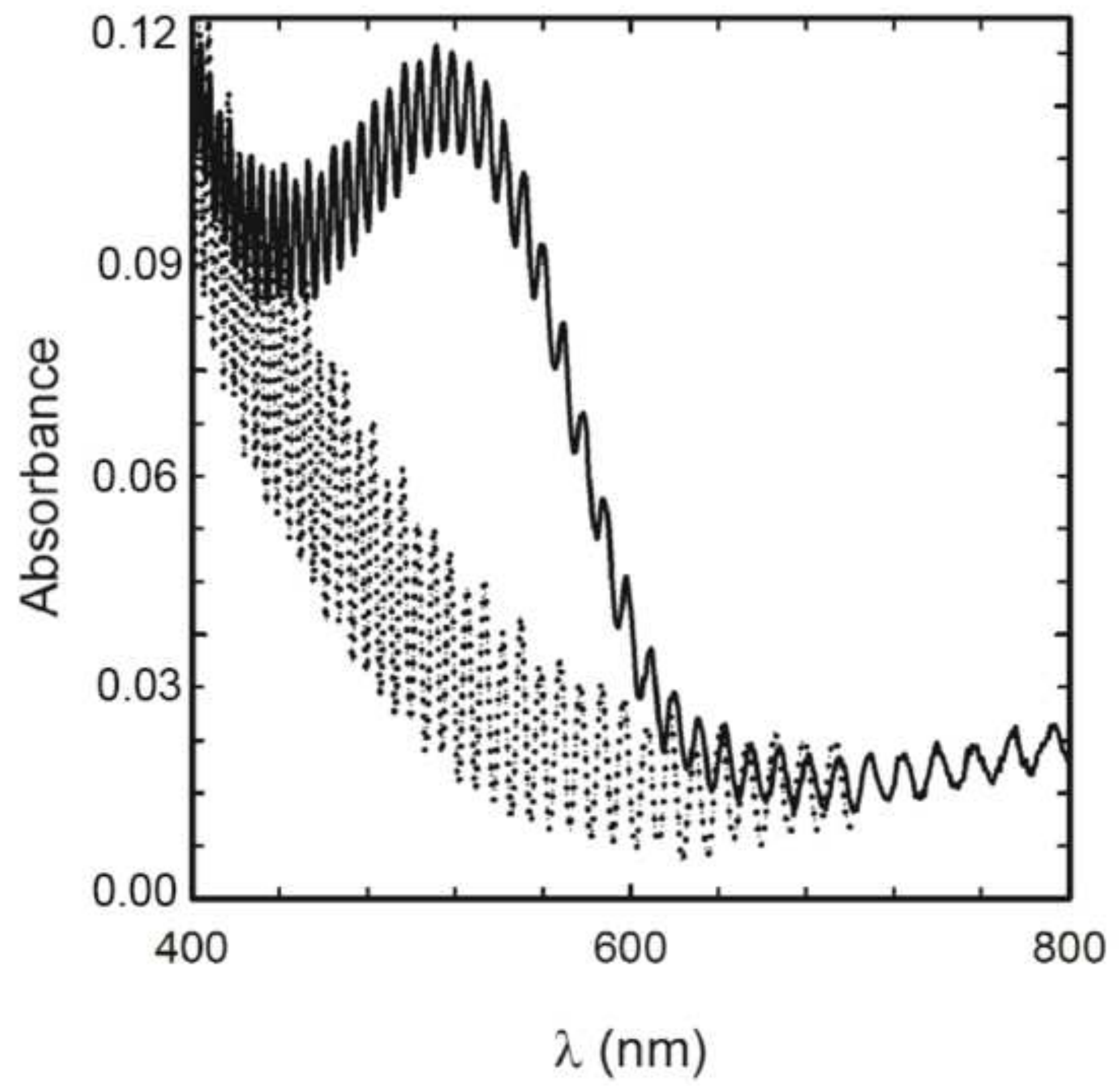

